\title{
Temporary threshold shifts in auditory sensitivity produced by the combined effects of noise and sodium salicylate
}

\author{
THOMAS L. BENNETT \\ Department of Psychology and Department of Physiology and Biophysics \\ and \\ R. JOHN MORGAN, PAULETTE MURPHY, AND LUCIAN B. EDDY \\ Department of Physiology and Biophysics \\ Colorado State University, Fort Collins, Colorado 80523
}

\begin{abstract}
Monaural chinchillas trained in behavioral audiometry, were used to test the hypothesis that the combined effects of noise and sodium salicylate intoxication produce a larger loss of hearing sensitivity or temporary threshold shift (TTS) than either agent alone can produce. The hypothesis was confirmed in that, while the TTS due to broad-band noise exposure of 85-dB intensity was $35 \mathrm{~dB}$ on the average, and the TTS, due to prolonged serum salicylate

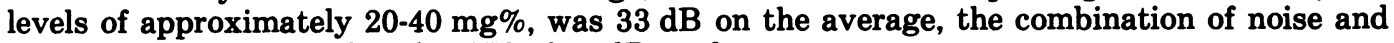
salicylate exposure produced a TTS of $55 \mathrm{~dB}$ on the average.
\end{abstract}

A special tragedy for humans is hearing loss. Two causes are prolonged exposure to high-intensity noise and the ingestion of ototoxic drugs, that is, drugs that produce a hearing loss. Exposure to noise can produce a hearing loss that is temporary or permanent. The longterm effects depend on the frequency spectrum of the noise, its intensity, and its duration, but even the traffic of automobiles and other vehicles in large cities is of sufficient intensity $(85 \mathrm{~dB})$ to produce a temporary loss of hearing sensitivity after a few hours of exposure (Miller, 1974).

Similarly, ototoxic durgs can produce either a temporary or permanent hearing impairment. Interestingly, common aspirin (sodium salicylate), when taken in high doses (as commonly done by humans suffering from arthritis), can produce a temporary decrease in hearing sensitivity (McCabe \& Dey, 1965; Myers \& Bernstein, 1965).

Extensive research has been conducted on the effects of exposure to noise and ototoxic drugs on hearing sensitivity. Research on the combined effects of these two agents has been limited (Falk, 1972). The available data, however, suggest that hearing is disrupted more when ototoxic drugs and noise are simultaneously administered than occurs from either agent alone (Dyal, Kokshanian, \& Mitchell, 1971; Gannon, 1969). These two studies examined the effects of noise and kanamycin, a drug that produces a permanent hearing loss. The present study continued this line of investigation by examining the combined effects of noise and salicylate exposure on hearing sensitivity.

\section{METHOD}

\section{Subjects}

Thirteen adult chinchillas, rendered monaural by surgical de- struction of the left cochlea (see Bennett, Morgan, Murphy, \& Eddy, 1978) were selected for this study. All of them were in excellent health and easy to handle. They were divided into two groups: Group A consisted of four males and three females; Group B consisted of two males and four females. These groups were similar with respect to pretreatment auditory thresholds and age.

\section{General Procedure}

The behavior training equipment and threshold testing procedures used in this experiment were described in our previous paper (Bennett et al., 1978). Pretreatment audiograms were obtained prior to the noise experiment (Group A), the salicylate experiment (Group B), and the noise-salicylate experiment (Group A).

\section{Noise Experiment}

This phase of the study was designed to measure the growth and decay of temporary threshold shifts (TTS) produced as a result of $85-\mathrm{dB}$ broad-band noise exposure. The chinchillas of Group A were exposed to the noise for $48 \mathrm{~h}$ in a small six-sided metal chamber $(105 \mathrm{~cm}$ long, $53 \mathrm{~cm}$ wide, and $28 \mathrm{~cm}$ deep) with 12-cm-thick insulated sides. Eight 8 -ohm speakers were mounted in series along the sides of the chamber and were driven by a Realistic stereo amplifier, Model SA-100C. A 6-h noise tape was made using a General Radio Company random noise generator, Type 1390-B. This generator produced a broad-band noise signal from 30 to $20,000 \mathrm{~Hz}$. The tape was played on a Realistic tape recorder, Model TR-88, and fed into the amplifier to drive the speakers. Three small wire cages $(38 \mathrm{~cm}$ long, $23 \mathrm{~cm}$ wide, and $20 \mathrm{~cm}$ high) were used to house the chinchillas during the noise exposure. Food and water were provided in each cage.

To follow the growth of the TTS, thresholds were measured during brief interruptions of the noise exposure. The effective duration of exposure was calculated by subtracting 3 min from the total elapsed time in noise for every $7 \mathrm{~min}$ spent out of noise (Ward, Glorig, \& Sklar, 1959). The growth of the TTS was followed by making threshold tests at $30 \mathrm{~min}, 1,2,4,8,12,24$, and $48 \mathrm{~h}$ into exposure. After termination of the noise, the animals were returned to their regular holding quarters to recover in relative quiet. The average ambient noise level in this room measured less than $50 \mathrm{~dB}$. Decay of the noise-induced TTS 
was followed by making threshold tests at $40 \mathrm{~min}, 3,8,12$, and $24 \mathrm{~h}$, and at $2,3,4,5,6$, and 7 days after the termination of noise.

\section{Salicylate Experiment}

The salicylate phase of the study was designed to measure the growth and decay of the TTS due to prolonged serum salicylate levels of approximately $20-40 \mathrm{mg} \%$. Each chinchilla of Group B was injected subcutaneously with a solution of sodium salicylate in physiological saline $(200$ or $100 \mathrm{mg} / \mathrm{cc})$ at the start of the experiment, $3 \mathrm{~h}$ later, and every $6 \mathrm{~h}$ thereafter, for a total duration of $84 \mathrm{~h}$. Individual doses of either 200 or $150 \mathrm{mg} / \mathrm{kg}$ were based on information obtained during a previous salicylate study (Eddy, 1974) and the blood levels of each animal, which were monitored during the experiment. This dosage resulted in serum salicylate levels being maintained at $20-40 \mathrm{mg} \%$. A modified version of the Trinder (1954) method as described by Caraway (1960) was used to determine serum salicylate levels.

To follow the growth of the salicylate-induced TTS, threshold tests were conducted $20 \mathrm{~min}, 1,3,6,12,24,36,48,54,60$, 72 , and $84 \mathrm{~h}$ after the initial injection. To follow the decay of the TTS, threshold tests were measured 6,12 , and $24 \mathrm{~h}$ and 2,3 , and 5 days after the last injection. At those intervals where multiple events occurred, a sequence was established such that the threshold test was performed, then a blood sample was taken, and finally the animal was injected with sodium salicylate.

\section{Noise-Salicylate Experiment}

Initially, each animal of Group A was exposed to only salicylate. Injections, doses, and serum salicylate levels were determined as described in the salicylate experiment section. The growth of the TTS due to salicylate was measured after $30 \mathrm{~min}$, $1,3,6,12,24$, and $36 \mathrm{~h}$. Thirty-six hours after the first salicylate injection, the animals were placed in the noise chamber and exposed to $85-\mathrm{dB}$ noise as well as continued salicylate injections. The last injection $(72 \mathrm{~h}$ ) was administered $6 \mathrm{~h}$ before the cessation of the noise.

Threshold tests to assess any change in the TTS resulting from the combined effects of these two agents were conducted after effective durations of $1,3,6,12,24$, and $42 \mathrm{~h}$. The noise was terminated after $42 \mathrm{~h}$ of exposure. As in the noise experiment, the effective duration of an exposure was calculated by the method used by Ward et al. (1959).

Each animal of Group A was exposed to the noise in the metal chamber described in the noise experiment section; however, there were two variations from the noise experiment: (a) The random noise generator was fed directly into the amplifier, and (b) smaller cages (21 cm long, $15 \mathrm{~cm}$ wide, and $15 \mathrm{~cm}$ high) were used to house the chinchillas in the chamber.

As in the salicylate experiment, at those intervals where multiple events occurred, a sequence was established such that the threshold test was performed, then a blood sample taken, and finally, the animals were injected with sodium salicylate. The decay of the TTS produced as a result of the combined effects of noise and sodium salicylate was followed by making theshold tests $1,3,6,12$, and $24 \mathrm{~h}$, and at $2,4,5,6$, and 7 days after the final termination of noise.

\section{RESULTS AND DISCUSSION}

\section{Preexposure Thresholds}

The preexposure thresholds measured at octave steps from .25 to $8.0 \mathrm{kHz}$ are displayed in Figure 1 . The solid line represents the mean thresholds for the seven chinchillas of Group A prior to the noise experiment. Each data point is the mean of 35 threshold values. The dashed line depicts the mean auditory

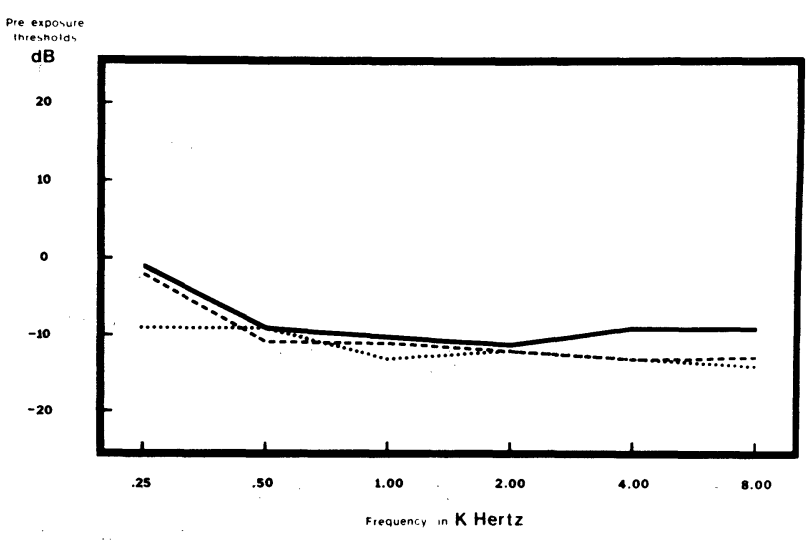

Figure 1. The preexposure thresholds measured at octave steps from .25 to $8.0 \mathrm{kHz}$ are displayed. Each data point is the mean of 35 threshold values. The solid line represents the average hearing threshold of Group A prior to the noise experiment. The dashed line depicts the mean auditory threshold from Group B prior to the salicylate experiment. The dotted line represents the average thresholds of Group A prior to the noise-salicylate experiments and were measured at least 45 days after the noise experiment.

thresholds of the six chinchillas of Group B prior to the salicylate experiment. Each data point is the mean of 30 threshold values. The dotted line represents the average thresholds of Group A prior to the noisesalicylate phase of the study. These thresholds were measured at least 45 days after the termination of the noise experiment. Each data point is the mean of 35 threshold values. Preexposure thresholds are highly similar prior to the exposures, the average threshold being approximately $-10 \mathrm{~dB}$.

\section{Noise Experiment}

Growth and decay of TTS at $.5,2.0$, and $8.0 \mathrm{kHz}$ are shown in Figure 2. These TTS are the result of exposure to broad-band noise at $85-\mathrm{dB}$ intensity. Each data point is the mean of threshold values for the seven chinchillas of Group A. The solid line represents the TTS measure at $.5 \mathrm{kHz}$, the dashed line represents the TTS at $2.0 \mathrm{kHz}$, and the dotted line depicts the TTS at $8.0 \mathrm{kHz}$. The growth of the TTS was found to be complete $24 \mathrm{~h}$ into noise exposure. The chinchilla's hearing sensitivity was reduced by approximately $35 \mathrm{~dB}$ on the average. A t test was performed to determine if this was a significant reduction of auditory sensitivity and was found to be significant $[\mathrm{t}(34)=5.8, \mathrm{p}<.01]$. The decay of the noiseinduced TTS was rapid and complete within $24 \mathrm{~h}$ after noise exposure.

\section{Salicylate Experiment}

The growth and decay of the TTS at 5.0 and $8.0 \mathrm{kHz}$ are shown in Figure 3. These TTS are the result of prolonged serum salicylate levels of $20-40 \mathrm{mg} \%$. Each data point is the mean of threshold values of the six chinchillas of Group B. The solid line represents the average 


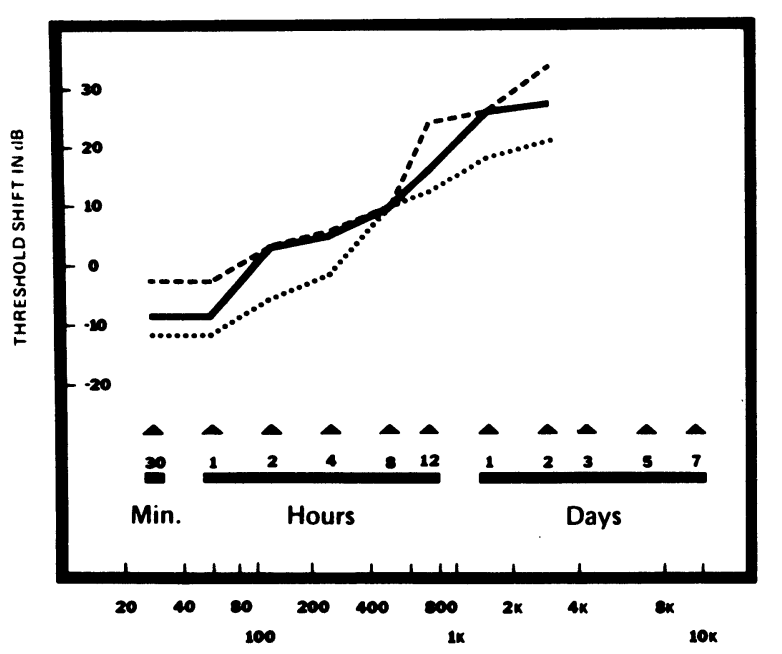

NOISE EXPOSURE TIME (MINUTES)

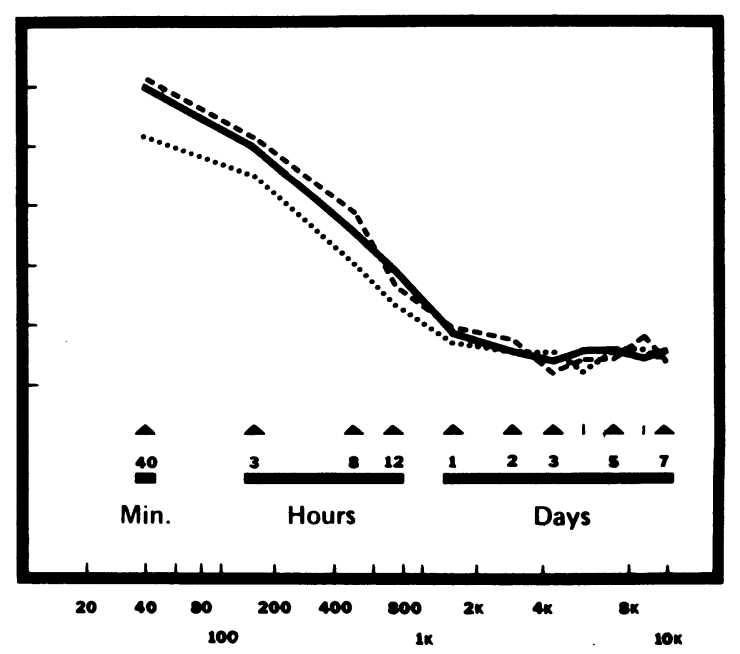

TIME (MINUTES) AFTER EXPOSURE

Figure 2. Growth and decay of noise-induced TTS at $.5,2.0$, and $8.0 \mathrm{kHz}$. Each data point is the mean of the threahold values for the seven chinchillas of Group A. The solid line represents the TTS measured at $.5 \mathrm{kHz}$. The dashed line depicts the average TTS measured at $2.0 \mathrm{kHz}$. The dotted line represents the TTS at $8.0 \mathrm{kHz}$.

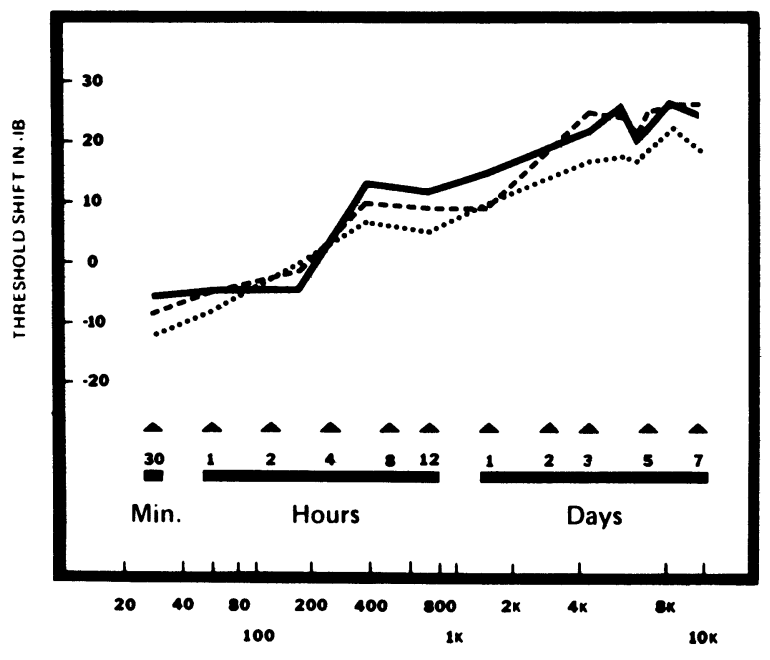

SALICYLATE EXPOSURE TIME IMINUTES)

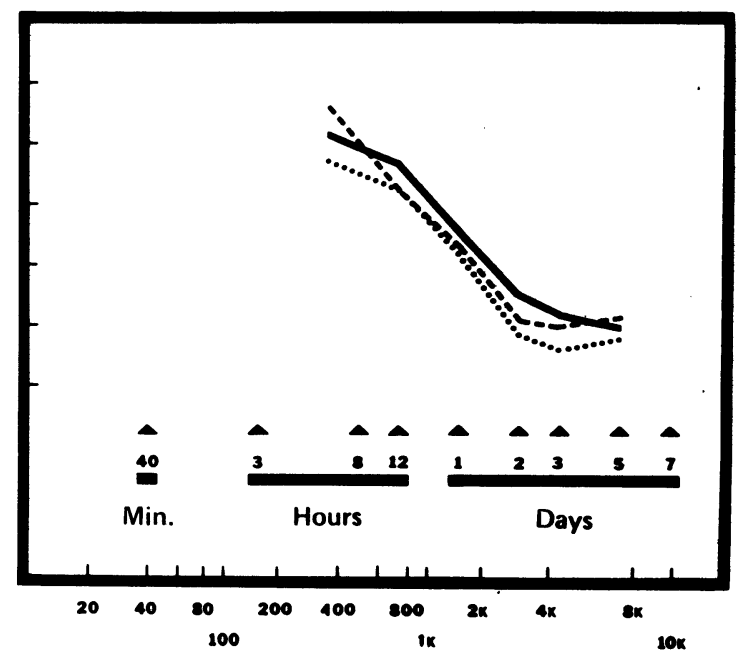

TIME (MINUTES) AFTER EXPOSURE

Figure 3. The growth and decay of the salicylate-induced TTS at $.5,2.0$, and $8.0 \mathrm{kHz}$. The dashed line shows the TTS measured at $2.0 \mathrm{kHz}$, and the dotted line depicts the TTS at $8.0 \mathrm{kHz}$. Each data point is the mean of threshold values of the six chinchillas of Group B.

TTS measured at $5.0 \mathrm{kHz}$, the dashed line depicts the average TTS measured at $2.0 \mathrm{kHz}$, and the dotted line represents the average TTS measured at $8.0 \mathrm{kHz}$. Hearing losss due to salicylate reached a maximum within $36-48 \mathrm{~h}$ of exposure. These serum salicylate levels resulted in a TTS of approximately $33 \mathrm{~dB}$ on the average. The $t$ value indicated that this was a significant reduction $[t(29)=5.5, p<.01]$. Decay of the TTS produced as a result of salicylate was relatively rapid and hearing sensitivity was back to preexposure levels within $48 \mathrm{~h}$ after cessation of the injections.

\section{Noise-Salicylate Experiment}

The TTS produced as a result of salicylate only $(36 \mathrm{~h})$ and subsequent combination of noise-salicylate exposure measured at $.5,2.0$, and $8.0 \mathrm{kHz}$ are shown in Figure 4. Each data point is the mean of threshold values of the seven chinchillas of Group A. The solid line represents the mean TTS measured at $.5 \mathrm{kHz}$, the dashed line depicts the TTS at $2.0 \mathrm{kHz}$, and the dotted line represents the TTS at $8.0 \mathrm{kHz}$. The growth of the salicylate-induced TTS was the result of prolonged serum salicylate levels of approximately $20-40 \mathrm{mg} \%$, as in the salicylate experiment. The hearing loss after $36 \mathrm{~h}$ of salicylate exposure was $30 \mathrm{~dB}$ on the average.

These serum salicylate levels and the $85-\mathrm{dB}$ noise exposure combined to produce the additional TTS increase shown in Figure 4. The hearing loss due to a combination of noise and salicylate reached a maximum in approximately $36 \mathrm{~h}$. At this time, the hearing sensi- 


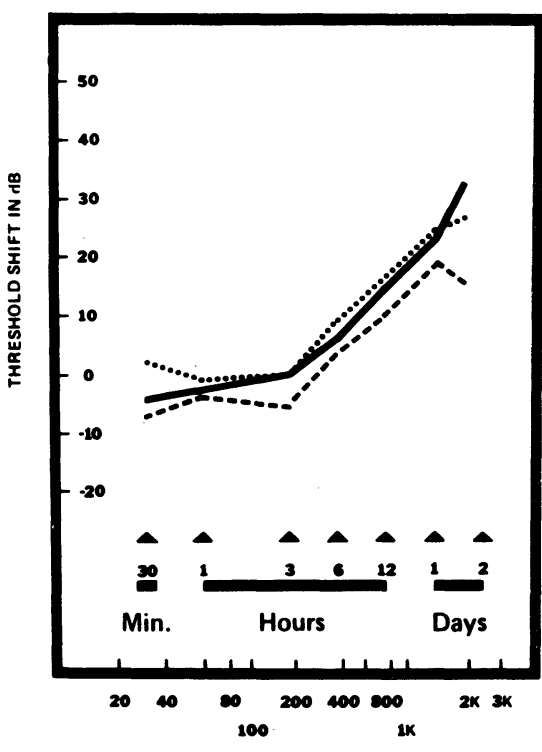

SALICYLATE EXPOSURE TIME (MIINUTES)

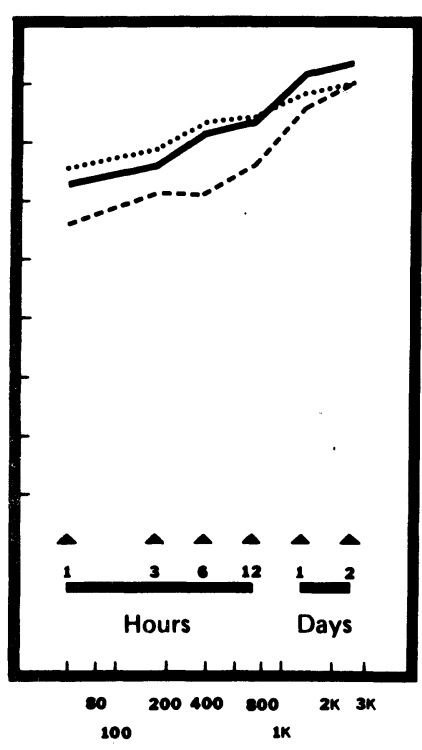

NOISE-SALICYLATE EXPOSURE TIME (MINUTES)

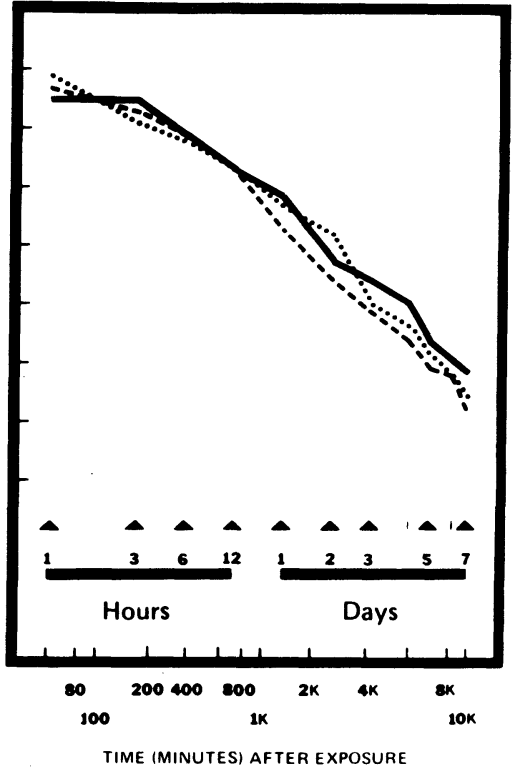

TIME (MINUTES) AFTER EXPOSURE

Figure 4. Growth and decay of the TTS at $.5,2.0$, and $8.0 \mathrm{kHz}$ produced as a result of salicylate-only and combination noise-salicylate exposures. The solid line represents the average threshold shifts produced at $.5 \mathrm{kHz}$, the dashed line displays the threshold shift at $2.0 \mathrm{kHz}$, and the dotted line depicts the threshold shifts at $8.0 \mathrm{kHz}$. Each data point is the mean of the threshold values for the seven chinchillas of Group A.

tivity of the chinchillas had been reduced by $55 \mathrm{~dB}$ on the average. A t test was performed which indicated that this reduction was significant $[\mathrm{t}(34)=9.6, \mathrm{p}<.01]$. The noise/salicylate-induced TTS did not disappear rapidly after the termination of exposure to these two agents. Three to 4 days were required before hearing sensitivity was near preexposure levels.

The results of this study suggest that the combined effects of prolonged noise exposure and salicylate intoxication result in a temporary threshold shift that is greater in magnitude than either agent alone can produce. The temporary hearing loss produced as a result of noise alone was $35 \mathrm{~dB}$.on the average, and that produced by salicylate was $33 \mathrm{~dB}$. However, the combination of these two agents produced a hearing loss of $55 \mathrm{~dB}$.

The recovery from the noise/salicylate-induced threshold shift was considerably slower than recovery from either the noise-induced or salicylate-induced hearing loss. While the decay of the noise-induced TTS was complete within $24 \mathrm{~h}$ and the decay of the TTS induced by salicylate was complete within $48 \mathrm{~h}$, the TTS produced as a result of the combined effects of these two agents required $3-4$ days before hearing sensitivity was near preexposure levels.

\section{REFERENCES}

Bennett, T. L., Morgan, R. J., Murphy, P., \& Eddy, L. B. The effect of unilateral surgical destruction of the cochlea on auditory sensitivity in the chinchilla. Bulletin of the Psychonomic Society, 1978, 12, 92-94.

CARAway, W. Microchemical method for blood analysis. Springfield, Ill: Charles C. Thomas, 1960.

Dyal, V. S., Kokshanian, A., \& Mrtchell, D. Combined effects of noise and kanamycin. Annals of Otology, Rhinology, and Laryngology, 1971, 80, 897-902.

EDDY, L. B. The combined effects of noise and sodium salicylate on hearing loss. Unpublished master's thesis, Colorado State University, 1974.

Falk, S. Combined effects of noise and ototoxic drugs. Environmental Health Perspectives, October 1972. Pp. 5-22.

Gannon, R. P. The occult effect of kanamycin on the cochlea. Excerpta Medica, 1969, 18, 98.

MCCABE, P., \& DEY, F. The effects of aspirin on auditory sensitivity. Annals of Otology, Rhinology, and Laryngology, 1965, 74, 312-325.

Miller, J. D. Effects of noise on people. Journal of the Acoustical Society of America, 1974, 56, 729-764.

Myers, E., \& Bernstein, J. Salicylate ototoxicity: A clinical and experimental study. Archives of Otolaryngology, 1965, 82, 483-493.

TRINDER, P. Rapid determination of salicylate in biological fluids. Biochemical Journal, 1954, 57, 301-303.

WARD, W. D., Glorig, A., \& SKLAR, D. Temporary threshold shifts from octave-band noise: Applications to damagerisk criteria. Journal of the Acoustical Society of America, 1959, 31, 522-528. 